\title{
GALAXY INTERACTIONS AND THE STIMULATION OF NUCLEAR ACTIVITY
}

\author{
Timothy M. Heckman \\ Department of Physics \& Astronomy \\ The Johns Hopkins University \\ and \\ The Space Telescope Science Institute
}

\section{INTRODUCTION AND DEFINITIONS}

The idea that interactions between galaxies can lead to enhanced galactic activity has a long and noble lineage. Since the early days of Baade and Minkowski (1954), through the seminal paper of Toomre and Toomre (1977) with its "stoking the furnace" imagery, to the similarly colorful and influential "feeding the monster" paper of Gunn (1979), and the landmark work by Larson and Tinsley (1978), this idea was elaborated upon and generalized. The pace of development of this hypothesis has accelerated greatly on both the observational and theoretical fronts during the 1980's, due in part to a growing perception that the AGN phenomenon could only be understood when the role of the environment in triggering or nurturing the activity was understood (cf. Balick and Heckman 1982). In recent years, the impact of the extragalactic IRAS database (on the observational side) and of the new generation of supercomputers and innovative software (on the numerical side) have led to an almost explosive growth in the number of papers written about this subject.

In the present review, I can not hope to discuss all the interesting papers (or even all the interesting ideas) that are germane. The reader is encouraged to consult four other recent reviews whose scopes overlap significantly with that of the present paper. Stockton (1990) has reviewed the connection between galaxy interactions and nuclear activity with an emphasis on the QSO phenomenon. Fricke and Kollatschny (1989) summarized the more general topic of the role of the environment in the AGN phenomenon. Kennicutt (this volume) reviews the effects of galaxy interactions on the stimulation of global starformation in galaxies and Schlosman (also this volume) discusses current theoretical ideas about how galaxy interaction might lead to the fueling of nuclear activity.

I would now like to loosely define some of the terms that I will be using throughout this review:

Nuclear - I will consider the "nucleus" to be the central kpc of a galaxy. This is largely a matter of observational convenience, in that most of the data available on nuclear activity in galaxies has been obtained with an effective aperture size of a kpc or so. Moreover, such phenomena as nuclear starbursts, the Narrow-Line- Region in classical AGN's, radio sources in Seyfert galaxies, and the thermal far-IR sources in many active galaxies have characteristic sizes of about a kpc (cf. Wilson and Heckman 1985; Telesco 1988).

Activity - By "activity" I mean luminosities that are significantly larger and/or high energy phenomena that are significantly stronger than could be sustained over a Hubble time by a normal population of stars. This definition explicitly includes "classical" AGN's (Seyfert galaxies, radio galaxies, QSO's) whose properties probably require the presence of a supermassive black hole (e.g. Rees 1984), optically-selected starburst nuclei (e.g. Balzano 
1893), and the IR-bright galaxies whose ultimate energy source is still controversial (e.g. Sanders et al. 1988a; Rieke 1988).

Interaction - I will include the full range of phenomena from mild "grazing" encounters to highly dissipative collisions (mergers). The nature (and limitations) of the evidence from which it is inferred that a given galaxy is "interacting" will be discussed in some detail in $\S$ III below.

\section{MOTIVATION}

In this section I would like to briefly discuss whether, quite apart from the observational evidence, there is a strong theoretical or heuristic motivation for investigating galaxy interactions as stimulators of nuclear activity in galaxies. As noted previously, detailed theoretical arguments concerning the mechanisms by which nuclear activity might be "fueled" are given elsewhere in this volume by Isaac Schlosman.

A. Nuclear Starburst/IR Galaxies

Nuclear starburst galaxies (a class in which I include the majority of IR-bright galaxies) require the presence of substantial interstellar matter within their central $\mathrm{kpc}$. The relatively low efficiency of stellar thermonuclear processes $\left(10^{-3} c^{2}\right)$, coupled with the severe energetic demands (ranging from $10^{59} \mathrm{ergs}$ for an M82-class starburst up to $10^{61} \mathrm{ergs}$ for "ultraluminous" starbursts) mean that interstellar masses of at least $10^{8}$ to $10^{10} \mathrm{M}_{\odot}$ are needed to fuel a nuclear starburst, even assuming $100 \%$ efficiency for the conversion of gas into high mass stars. In a growing number of cases, mm-wave interferometric maps of the molecular gas provide direct observational evidence for such material (cf. Sanders et al. $1988 \mathrm{~b}$ and references therein).

As Larson (1987) and others have argued, the surface mass density of the cold interstellar matter in starburst nuclei is so high (typically $1000 \mathrm{M}_{\odot} \mathrm{pc}^{-2}$ ), that the growth time for gravitational instabilities is extremely short (a million years). Moreover, the timescales for gas depletion via star-formation and/or supernova-driven outflows are also short compared to typical galaxian dynamical timescales $\left(10^{7}\right.$ to $10^{8}$ years vs. $10^{8}$ to $10^{9}$ years respectively). Larson therefore argues that since the gas that fuels the starburst must be assembled faster than it is consumed, and since the gas has a mass comparable to the entire mass of the ISM in a normal galaxy, powerful nuclear starbursts can only occur when some process allows a substantial fraction of the ISM of a galaxy to flow inward by an order-of-magnitude in radius at velocities $\gg$ than the typical non-circular velocities in ordinary disk galaxies $\left(\mathrm{v}_{\text {infall }} \gg 10 \mathrm{~km} / \mathrm{sec}\right.$ ). By way of illustration, Larson emphasizes that the collapse of a self-gravitating system implies a maximum infall rate 25 ( $\mathrm{v}_{\text {infall }} / 50$ $\mathrm{km} / \mathrm{sec})^{3} \mathrm{M}_{\odot} /$ year. This can be compared to typical estimated star-formation rates of 10 to $100 \mathrm{M}_{\odot} /$ year in starburst nuclei. To summarize, the fueling of a starburst nucleus requires a mechanism that can induce non- circular motions that are both large in amplitude and involve a substantial fraction of the ISM of the galaxy.

Thus, galaxy interactions are a very attractive mechanism for triggering nuclear starbursts for two primary reasons: 1 ) nuclear starbursts involve interstellar masses $\approx$ the mass of the galaxian ISM (meaning that they probably require transporting material in from large radii where tidal forces should be most important), and 2) nuclear starbursts require that the material be moved inward by a modest radial factor ("only" an orderof-magnitude!), an effect that has been observed in several recent N-body simulations by 
Noguchi (1988) and Hernquist (1989) of interactions between galaxies with dissipative particles (an "ISM").

B. Classical AGN's

The motivation for investigating galaxy interactions as stimulators of low-luminosity AGN's is less clear than in the case of the starburst nuclei (at least within the standard "supermassive-black-hole-plus-accretion" paradigm for AGN's):

First, the high efficiency of accretion into a relativistic potential ( $\left.20 \% c^{2}\right)$ implies that only modest accretion rates are needed to power even a bright Seyfert nucleus (e.g. 0.01 $\mathrm{M}_{\odot} /$ year for the nucleus of NGC 1068 , or a total accreted mass of $10^{6} \mathrm{M}_{\odot}$ for a nominal AGN lifetime of $10^{8}$ years). Moreover, there is as yet little direct observational evidence that low-luminosity AGN's are characteristically accompanied by large nuclear masses of interstellar gas. For example, the mass of $\mathrm{H} \mathrm{II} \mathrm{in} \mathrm{the} \mathrm{kpc-scale} \mathrm{Narrow-Line-Region} \mathrm{is}$ only $10^{3}$ to $10^{6} \mathrm{M}_{\odot}$ in typical Seyferts. Of course, the total interstellar mass in this region may be much larger than this. Meixner et al. (1990) have recently observed three Seyfert galaxies with strong mm-wave $\mathrm{CO}$ emission and find bright nuclear $\mathrm{CO}$ sources in two of them. In these two cases (NGC 3227 and NGC 7469 - both interacting galaxies), the implied molecular masses within the central few hundred pc are $\approx 10^{8} \mathrm{M}_{\odot}$. It is clearly important to determine whether such structures are commonly present in typical Seyfert nuclei.

Second, the region of energy extraction in the standard model of an AGN is many orders-of-magnitude smaller than the characteristic dimensions of the region susceptible to tidal forces during a galaxy encounter. That is, to explain the transport of material inward to the accretion disk, one must appeal to processes in addition to those directly resulting from a galaxy interaction.

Thus, galaxy interactions may be neither necessary nor sufficient for triggering lowluminosity AGN's because: 1) the fueling of low-luminosity AGN's probably involves masses « the mass of the galaxian ISM (meaning that they would not require transporting material in from the large radii where tidal forces should be most important), and 2) AGN's require that the material be moved inward to radii that are many orders-ofmagnitude smaller than the region that is directly effected by tidal forces during a galaxy interaction.

The situation may be quite different for the very powerful AGN's (particularly the most powerful QSO's and radio galaxies that exist almost exclusively at large redshifts). Again, within the standard AGN paradigm (Eddington-limited accretion), the most pow-

erful QSO's require black hole masses of $10^{9}-10^{10} \mathrm{M}_{\odot}$ (the mass of powerful starburst nuclei and a substantial fraction of the mass of the ISM). The formation of such a black hole surely involves spectacular dynamical processes, but the need to invoke (proto?)galaxy interactions is not clear.

\section{TYPES OF EVIDENCE AND SOME CAVEATS}

\section{A. Galaxy Morphology}

One of most frequently employed criteria for classifying a galaxy as "interacting" is morphological peculiarity. The greatest advantage of this criterion (apart from the relative ease of obtaining optical images) is that morphological peculiarities can provide evidence that a severe interaction has occurred within the last Gigayear even when there is no 
presently obvious candidate for the perturbing galaxy (i.e. after the perturber has merged with the galaxy in question or moved several hundred $\mathrm{kpc}$ away from it). However, using morphological peculiarities to link galaxy interactions to the fueling of nuclear activity does have some potential pitfalls, as I now describe in the form of four propositions.

1. The most convincing evidence that morphological peculiarities in active galaxies have arisen via tidal forces is to show that the peculiarities are present in the old ( $i$ Gigayear) stellar population (i.e. detect what we might call "Crimson Tides" in honor of our Alabama hosts). The least convincing evidence is when such structures are present only in the gas.

My point here is that when one is dealing with a galaxy having a highly active nucleus, the possibility of morphological peculiarities that have arisen through hydrodyamical processes driven by the nuclear activity must be considered. That is, the nuclear activity may be able to could propel gas outward and possibly even induce star-formation in this compressed, accelerated material (cf. Williams and Christiensen 1985).

One place where such processes are probably operating is in the high-redshift radio galaxies. Their distorted "multi-modal" optical morphology and high-velocity non-circular gas motions were initially cited as strong evidence that they were collisions or mergers of gas-rich galaxies (e.g. Djorgovski 1987). However, these peculiar structures were subsequently shown to be preferentially aligned with the radio source axis (McCarthy et al. 1987; Chambers, Miley, and van Breugel 1987), and they are therefore more likely to be the result of the interaction between the radio plasma and the inter-stellar/circum-galactic medium ("jet-induced starbursts", cf. Chambers 1989; Begelman and Cioffi 1989).

Closer to home, there is a mounting body of evidence that the kinetic energy supplied by massive stars and supernovae in the nuclei of starburst galaxies can drive galaxy-scale outflows (see Heckman, Armus, and Miley 1990 and references therein). In extreme cases (the "ultraluminous" IR galaxies) these "superwinds" can produce galaxy-scale regions in which the local gas pressures $\left(P / k>10^{6} \mathrm{~K} \mathrm{~cm}^{-3}\right)$ are orders-of-magnitude larger than normal interstellar pressures. The superwinds are clearly shaping the morphology and kinematics of the ionized gas, and might conceivably be responsible (via induced starformation) for at least some of the structural peculiarities commonly visible in optical continuum images of IR-luminous galaxies.

2. It is important to have a good control sample to determine what fraction of non-active galaxies would be classified as morphologically peculiar. At the very least, the criteria used to classify a galaxy as "peculiar" need to be as quantitative and reproducable as possible.

This is a tired and trite truism that is not always adhered to in investigations of the link between morphological peculiarities and nuclear activity (including some of the author's own efforts in this regard!). Part of the problem is that it is not always clear that an appropriate comparison sample can be defined (e.g. what is the proper comparison sample for high-redshift QSO's?).

3. Not all peculiar structures have morphologies that can be readily explained as tidal features.

While numerical simulations of galaxy interactions have been remarkably successful in reproducing the kinds of peculiar structures that populate the Arp Atlas, there are some counter-examples that are very difficult to ascribe to tidal forces (see the recent review by 
Keel 1987). The most spectacular and well- known example is probably NGC 1097 with its multiple radial "jets", some of which show remarkable right angle bends.

4. Some apparent morphological peculiarities are simply the result of the overlap of the images of two or more structurally normal galaxies.

Some very nice illustrations of this can be found in a recent paper by Lauer (1989). His "deconvolutions" of images of multiple-nucleus galaxies at the centers of galaxy clusters showed that in only about half of the cases were the individual galaxian sub-components actually distorted.

B. Proximity

This type of evidence for an interaction ("guilt by association") is typically provided by counting the number of galaxies within several galaxy diameters of the active galaxy (with the neighboring galaxies often weighted by their size and/or proximity to the active galaxy). This scheme is designed to quantify the likely importance of tidal stresses suffered by a galaxy within the last Gigayear (i.e. within the last few galaxy dynamical timescales). Some rather obvious caveats:

1. It is important that the explicit and implicit selection criteria used in generating the sample of active galaxies be fully understood and that this understanding then be used to create a suitable comparison sample of non-active galaxies.

2. The galactic background should be well-determined so that the counts of neighbors can be statistically corrected for contamination by foreground/background galaxies, or the background should clearly be shown to be negligible. This is particularly important when the active galaxies under investigation are at high redshifts and when the lower end of the companion galaxy luminosity function is being probed (cf. Smith and Heckman 1990a).

C. Kinematic Evidence

Kinematic evidence for an interaction or past merger includes the detection of noncircular motions at $v \gg 10 \mathrm{~km} / \mathrm{sec}$ and the discovery of kinematically-distinct subsystems in a galaxy. Again, some of the potential pitfalls in linking kinematic peculiarities to galaxy interactions are:

1. Peculiar kinematic structures in the gas may arise through gravitational forces or through hydrodynamical ones associated with the nuclear activity. This is essentially the same argument as given above for morphological peculiarities. Stellar-dynamical investigations of active galaxies are less ambiguous in this regard, but far more difficult observationally.

2. Investigations of the kinematics of gas based on emission- lines are subject to ambiguity in the sign of any radial motions that are detected. While infalling gas could be linked to the cause of nuclear activity, outflowing gas is more plausibly ascribed to the effect. Absorption-line studies of gas seen in front of active nuclei are clearly superior in this regard, even though they probe only a single line-of-sight.

3. Kinematic sub-structures in galaxies may be very long-lived, and so provide no evidence that an interaction or merger has occurred recently enough to plausibly link it to the triggering of nuclear activity. For example, the counter-rotating stellar cores found in some nearby elliptical galaxies could have formed a Hubble time ago (cf. Franx and Illingworth 1989). Similarly, if elliptical galaxies are triaxial, it is possible for a stable (longlived) gaseous disk to exist that is strongly warped and/or misaligned with the principal 
photometric axes of the galaxy (see van Albada, Schwarzschild, and Kotanyi 1982).

\section{EVIDENCE LINKING INTERACTIONS TO NUCLEAR ACTIVITY}

\section{A. Infrared/Starburst Galaxies}

My emphasis here will primarily be on galaxies with high rates of star formation in their nuclei and/or galaxies whose IR luminosities are large compared to typical galaxies $\left(\mathrm{L}_{\mathrm{IR}}>10^{44} \mathrm{erg} / \mathrm{sec}\right)$. The detailed consideration of whether relatively mild enhancements in the global rate of star-formation are induced by galaxy interactions can be found in the review by Rob Kennicutt elsewhere in this volume. It is beyond the scope of this paper to review the evidence that the majority of IR-luminous galaxies are indeed powered by starformation occurring within their central- most kpc (see recent reviews by Soifer, Houck, and Neugebauer 1987 and Telesco 1988).

Investigations of the link between high rates of nuclear star formation and galaxy interactions can be divided into two principal categories: 1) determination of the nuclear star-formation rates in samples of known interacting galaxies (e.g. pairs of galaxies or galaxies in the Arp Atlas) and 2) determination of the optical morphology and/or local environment of galaxies selected to have abnormally high star-formation rates (e.g. strong IRAS sources). The former studies usually provide the best statistics about low- level activity, while the latter type provide information about the relatively rare, powerful nuclear starbursts.

i) Star-Formation Rates in "Known" Interacting Galaxies

The star-formation rates in pairs of galaxies have been compared to those in isolated galaxies using $\mathrm{H} \alpha$ luminosities and equivalent-widths, mid- and far-IR luminosities, and nonthermal radio powers (Bushouse 1986,1987; Bushouse, Lamb, and Werner 1987; Cutri and McAlary 1985; Hummel 1981; Keel et al. 1985; Kennicutt et al. 1987; Sulentic 1976,1990; Stocke 1978). Taken together, these many studies lead to the following conclusions (see Kennicutt's review for more details): 1 . The enhancement in the global star-formation rate in the pairs is modest (increased by an average of $50-100 \%$ relative to the isolated galaxies), but statistically significant. 2. The enhancement in the nuclear star-formation rate is stronger (by an average factor of at least three compared to isolated galaxies). 3. There is no strong dependence of the amount of enhanced star-formation on the pair separation.

Samples of "strongly interacting" galaxies (usually selected from the Arp Atlas on the basis of morpholgical evidence that they are undergoing an interaction) have been investigated in the IR, optical, and radio by Bushouse $(1986,1987)$, Bushouse, Lamb, and Werner (1988), Carter et al. (1988), Heckman (1983), Hickson et al. (1989), Joseph and Wright (1985), Joseph et al. (1984), Keel et al. (1985), Kennicutt et al. (1987), Larson and Tinsley (1978), Lonsdale, Persson, and Mathews (1984), and Sulentic (1976). These represent a more heterogeneous set of galaxies than those in the "pairs" sample (and present a greater challenge in properly constructing a comparison sample). However, they are important to investigate because they are undergoing stronger and more damaging tidal encounters than the typical galaxy in the "pairs" samples. The principal results of the papers cited above can be summarized as follows: 1 . The data imply that the global starformation rates in these galaxies are enhanced relative to "normal" disk galaxies by average factors of at least two. 2. The enhancement in star-formation is most pronounced in the 
nuclei of the galaxies (at least as evidenced by the distribution and brightness of the $\mathrm{H} \alpha$ line emission). 3. The "Toomre-type" candidates for on-going mergers of two major disk galaxies (e.g. Toomre 1977) are exeptionally luminous in the IR and radio. I will shortly discuss this result and its implications. 4. Elliptical galaxies with shells are unusually likely to have strong Balmer absorption-lines compared to normal ellipticals (Carter et al. 1988). The Balmer lines are considerably stronger than those in spirals with the same broad-band colors, so these galaxies can not be simply interpreted as merged spirals with normal stellar populations. Rather, they represent evidence for fossil starbursts $\left(10^{8}\right.$ to $10^{9}$ years ago), possibly triggered by the capture event that produced the shells. Schweizer and Seitzer (1990) have reached similar conclusions for ellipticals with very subtle fine-scale substructure.

While the case for the stimulation of star-formation by galaxy interactions seems clearly established, there are several points I would like to emphasize concerning the reliability or meaning of the "enhancement factors" summarized above.

First, the projected aperture sizes (in square parsecs) used to measure the $\mathrm{H} \alpha$ and the near/mid-IR properties for the paired or interacting galaxies are typically larger (sometimes by substantial factors) than for the "control" galaxies. This is usually because the latter data were gathered from the literature and had been obtained for the nearest, brightest "normal" galaxies (i.e. galaxies significantly nearer than typical Arp Atlas galaxies). In many cases, honest efforts to estimate, correct for, or otherwise mitigate against the potential biases introduced by these aperture mismatches have been made. For example, the $\mathrm{H} \alpha$ equivalent width (which is far less aperture dependent than the $\mathrm{H} \alpha$ luminosity) is frequently employed in the comparisons of the galaxy samples. Nevertheless, the aperture effect sometimes makes it difficult to compare the interacting and "normal" galaxies in a detailed and rigorously quantitative sense.

Second, in most cases, the star-formation rates in the interacting (or paired) samples and "control" samples are compared after being normalized to the blue luminosity of the galaxy. This is designed to cancel out (statistically) any effect as trivial as big and optically-bright galaxies being bright at other wavelengths as well. However, the origin of the blue light in an interacting galaxy is a little unclear - such galaxies could have their blue luminosities boosted by enhanced star-formation or depressed by increased internal extinction. A better normalization would be to the mass of the galaxy (difficult to obtain) or possibly to the luminosity at $\mathrm{J}$ or $\mathrm{H}$ where the effects of dust and young stars should be greatly diminished. A related difficulty (particularly for the often badly-distorted galaxies in the Arp Atlas) is in selecting the proper mix of Hubble types in the comparison sample. This problem is acute because of the well-known correlation between Hubble type and starformation. One really wants to know the Hubble type of the interacting/peculiar galaxy before it interacted!

Third, it is important to note that there is certainly no one-to-one match between interactions and enhanced star-formation. There is a substantial overlap in the estimated star-formation rates between the samples of interacting and normal galaxies. Indeed, Bushouse (1986) emphasized that the nuclei of about $30 \%$ of his strongly interacting galaxies showed no optical evidence for recent star-formation (see also Joseph et al. 1984).

Finally, the translation of IR luminosities, nonthermal radio powers, recombination- 
line luminosities, etc. into estimates of the star-formation rate are fraught with many uncertainties (e.g. the importance of dust, the nature of the IMF and the time-history of the star-formation, the potential roles of the old stars or an AGN, the uncertain physics of the origin of the radio synchrotron emission, etc.). These problems are particularly vexing in the nuclei of galaxies. Multi-wavelength investigations are essential to attack these problems.

Before concluding this subsection, I want to just briefly explore some of the implications of the strongly enhanced radio and IR luminosities of the galaxies that Toomre (1977) selected as the best local examples of two major disk galaxies in the process of merging to form an elliptical galaxy. The radio properties of this kind of galaxy were explored by Heckman (1983) and the mid-IR properties by Joseph and Wright (1985). I have listed in Table 1 the optical and far-IR properties of the 10 Toomre disk-disk merger candidates with redshifts known to me. As a class, these galaxies are characterized by large far-IR

\section{TABLE 1}

IR and Optical Properties of Toomre's Disk-Disk Merger Candidates

Galaxy

NGC 4038/9 1650

NGC $4676 \quad 6600$

NGC $7592 \quad 7300$

NGC 6621/2 6250

NGC $3509 \quad 7650$

NGC $520 \quad 2150$

NGC $2623 \quad 5450$

NGC $3256 \quad 2900$

NGC $3921 \quad 6000$

NGC $7252 \quad 4750$
(3)

(4)

(5)

$\begin{array}{ccc}\log \mathrm{L}_{\mathrm{FIR}} & \mathrm{L}_{\mathrm{FIR}} / \mathrm{L}_{\mathrm{B}} & \mathrm{F}_{100} / \mathrm{F}_{60} \\ 10.5 & 1.8 & 1.2 \\ 10.5 & 1.7 & 1.1 \\ 11.1 & 3-5 & 0.8 \\ 10.9 & 2.0 & 1.1 \\ 10.4 & 0.6 & 1.8 \\ 10.6 & 1.7 & 0.9 \\ 11.3 & 11 & 0.7 \\ 11.3 & 4.6 & 0.8 \\ 10.0 & 0.2 & 1.2 \\ 10.5 & 0.6 & 1.0\end{array}$

NOTES

Col. 3 - The $\log$ of the far-IR (40 to $120 \mu$ ) luminosity (in $\mathrm{L}_{\odot}$ ) calculated from the "FIR" flux given in Cataloged Galaxies and Quasars Observed with IRAS assuming $\mathrm{H}_{0}=75 \mathrm{~km}$ $\mathrm{sec}^{-1} \mathrm{Mpc}^{-1}$.

Col. $4-\mathrm{L}_{\mathrm{B}}$ is calculated using $\mathrm{M}_{\mathrm{B}, \odot \odot}=5.48$ and apparent $\mathrm{B}$ magnitudes $\left(\mathrm{B}_{T}\right.$ scale) for the galaxies taken from the Revised Shapley Ames Catalog, the Uppsala General Catalog of Galaxies, and the Second Reference Catalog of Bright Galaxies. They have been corrected for foreground Galactic extinction, but not for extinction internal to the galaxies. These references give no B magnitude for NGC7592, so I used a rough estimate based on $\mathrm{L}_{\mathrm{B}}$ for the other galaxies.

Col. 5 - The ratio of the IRAS fluxes (P) at $100 \mu$ and $60 \mu$. Note that Bothun, Lonsdale, and Rice (1989) argue that the far-IR luminosity can be used to estimate the star-formation rate for $\mathrm{F}_{100} / \mathrm{F}_{60}<1.5$ in my units. 
luminosities (average $\mathrm{L}_{\mathrm{FIR}}=8 \times 10^{10} \mathrm{~L}_{\odot}$ ), large ratios of IR to optical luminosity (average $\mathrm{L}_{\mathrm{FIR}} / \mathrm{L}_{\mathrm{B}} \approx 3$ ), and "warm" far-IR colors (average ratio of $\mathrm{S}$ at $60 \mu$ and $100 \mu \approx 1.0$ ). This suite of characteristics suggests that they are forming massive stars at rates well in excess of those in typical bright spiral galaxies (cf. Bothun, Lonsdale, and Rice 1989). Toomre has argued that his set of galaxies represent various evolutionary stages in the merging process, from the NGC 4038/39 system (two well-separated galaxies) to NGC 7252 (a candidate "proto-elliptical" with a single main body plus two well-defined tidal tails). The enhanced IR emission is seen throughout the sample, with no obvious trend along Toomre's suggested evolutionary sequence. This suggests that the duration of the enhanced star-formation induced by the merger process is at least as long as the timescale for a merger ( $>$ a few $\times 10^{8}$ years).

The average far-IR luminosity of the disk-disk merger candidates implies a starformation rate of $50 \mathrm{M}_{\odot}$ per year, assuming a Salpeter IMF and a constant star-formation rate (cf. Hunter et al. 1986). If this lasts for 300 Megayears, the implied total mass of stars formed as a result of the merger is $1.5 \times 10^{10} \mathrm{M}_{\odot}$. In most well-studied IR-luminous galaxies, much/most of the star-formation is occurring within a radius of a $\mathrm{kpc}$ of the nucleus in a massive disk of molecular gas (cf. Sanders et al. $1988 \mathrm{~b}$ and references therein). If this is the case in the merger candidates, and if Toomre's hypothesis concerning the formation of elliptical galaxies is correct, then the aftermath of the merger will be a galaxy whose global structure and dynamics is that of a bright elliptical, but whose core contains a rapidly rotating "post-starburst" disk with a mass of $10^{10} \mathrm{M}_{\odot}$ and a size of about a kpc. Even after several Gigayears, when this disk might no longer be photometrically obvious, it could be recognized through its kinematic signature. If a substantial fraction of elliptical galaxies formed this way, might not such dynamically-distinct cores be common? The recent discovery of kinematically-distinct cores in bright elliptical galaxies is extremely interesting in this regard (Franx and Illingworth 1988; Jedrzejewski and Schecter 1988). The large fraction of "shelled" ellipticals with post-starburst nuclear spectra may also be relevant (Carter et al. 1988).

ii) The Morphology and Environment of IR and Starburst Galaxies

The bulk of the data concerning either the optical morphology or the local environment of galaxies with known/suspected nuclear starbursts concerns galaxies selected on the basis of strong far- IR emission (cf. Armus, Heckman, and Miley 1987,1990; Fairclough 1986; Lawrence et al. 1989; Sanders et al. 1988a). Such samples are easily generated, but suffer from some ambiguity of interpretation (as noted above, there is still some controversy about what fraction of far-IR-luminous galaxies are indeed powered by nuclear starbursts vs. a "buried QSO" or even vs. an unusually heavily obscured "normal" stellar population). The imaging surveys listed above document a trend towards an increased fraction of morphologically peculiar galaxies in systems of higher IR luminosity:

$>$ About $10 \%$ of the galaxies with IR luminosities ranging from $10^{10}$ to $10^{11} \mathrm{~L}_{\odot}$ are morphologically peculiar. This is not significantly different from the fraction of morphologically peculiar galaxies in randomly-selected samples of field galaxies (cf. Lawrence et al. 1989; Armus, Heckman, and Miley 1987). It is important to realize that this luminosity range brackets the "knee" in the IR luminosity function of galaxies: thus, the galaxies that dominate IR-flux- limited samples do not have a strongly abnormal incidence rate of peculiar 
optical morphologies.

$>$ About $50 \%$ of the galaxies with IR luminosities in the range $10^{11}$ to $10^{12} \mathrm{~L}_{\odot}$ are classified as morphologically peculiar, and the fraction reaches essentially $100 \%$ for the rare "ultraluminous" galaxies with $\mathrm{L}_{\mathrm{IR}}>10^{12} \mathrm{~L}_{\odot}$.

$>$ It is a peculiar morphology (presumably indicative of severe tidal damage - but see §IIIA above) rather than the mere presence of companion galaxies that seems to be the key characteristic that sets apart the powerful IR galaxies $\left(L_{I R}>10^{11} L_{\odot}\right)$ from more normal galaxies. About half of the powerful IR galaxies studied by Armus, Heckman, and Miley $(1987 ; 1990)$ are peculiar in optical morphology, but are not observed to be obviously interacting with any other galaxy. In more detail, Lawrence et al. have classified their IRselected and control (field galaxy) samples into six interaction classes ranging from 0 (no companions, normal morphology), 1-4 (normal morphology, but progressively brighter and closer companions), through 5 and 6 (galaxy pair with peculiar morphology and "merger" = single galaxy with peculiar morphology). Interestingly the ratio of powerful IR galaxies in classes 1-4 to those in Class 0 is similar to the same ratio for the field galaxies. The powerful IR galaxies are distinguished from the field galaxies by the relatively large fraction in Classes 5 and 6 (morphologically peculiar).

$>$ The incidence rate of multiple nuclei (visible in high resolution near-IR images) is at least $50 \%$ in the "ultraluminous" IR galaxies (Carico et al. 1990; Graham et al. 1990; Illingworth et al. in preparation). The typical inter-nuclear separation is only a few kpc, and the timescale for the final coalesence of the nuclei via dynamical friction is then only $10^{8}$ years. This suggests that the ultraluminous phase corresponds to the final $10^{8}$ years of a merger. This age estimate is consistent with other estimates of the duration of nuclear starbursts.

There has been less analysis of the possible role of galaxy interactions in the opticallyselected samples of nuclear starburst and Blue Compact Dwarf ("BCD" or "HII") galaxies (cf. Balzano, 1983; Melnick 1987; Salzer 1987). Both Campos-Aguilar and Moles (1990) and Melnick (1987) find that only a minority (20-30\%) of the BCD's are members of interacting systems. Brinks (1990) has suggested (based on $21 \mathrm{~cm}$ HI maps of two BCD's) that they may be triggered by collisions of $\mathrm{HI}$ clouds rather than collisions of galaxies. CamposAguilar and Moles (1990) find that all the nuclear starburst and irregular blue galaxies in their sample have massive companion galaxies. Finally, Condon et al. (1982) have emphasized the role of galaxy interactions in triggering starbursts in a radio-selected sample of spiral galaxies (a sample which was subsequently discovered to contain an abundance of now-famous IRAS galaxies).

\section{B. Seyfert Galaxies}

As in the case of starburst/IR galaxies, studies of the link between galaxy interactions and the Seyfert phenomenon fall into two primary categories: comparisons of the optical morphology and local environments of known Seyfert galaxies to those of non- Seyferts and comparisons of the strength and incidence rate of Seyfert nuclei in samples of interacting and non-interacting galaxies.

i) The Morphology and Environment of Known Seyfert Galaxies

There have been several recent observational investigations of the local environments of large samples of Seyfert galaxies. The studies have reached somewhat different conclu- 
sions, and therefore I will describe them in some detail.

Dahari (1984) determined the fraction of Seyferts with close (ithree galaxy diameters) companion galaxies. His sample included 103 Seyfert galaxies, with Seyferts in rich clusters being specifically excluded. His control sample consisted of randomly- selected galaxies in the same general fields as the Seyfert galaxies whose diameters were $75-150 \%$ those of the Seyferts. After correcting for the background using estimates based on the ShaneWirtanen Catalog, he found that $15 \%$ of the Seyferts had close companions vs. $3 \%$ of the control sample (a significant difference). He subsequently (Dahari 1985) obtained redshifts for 33 of the Seyfert companions, and found no correlation between either radio continuum power or $\mathrm{H} \alpha$ luminosity and any measure of the importance of tidal interaction (e.g. pair separation, relative size of companion, redshift differences). The Seyferts with close companions did not differ in either radio power or $\mathrm{H} \alpha$ luminosity from isolated Seyferts at a statistically significant level.

Dahari and DeRobertis (1988) investigated a sample of 194 Seyfert galaxies, searching for relationships between tidal interactions and the properties of the nuclei. They found no statistically significant differences between the Seyferts with and without companions, except that the type 2 Seyferts with companions had excess infrared and radio-continuum emission (by an average factor of 5) compared to those without. Dahari and DeRobertis suggest that this excess IR and radio emission may be due to enhanced star-formation rather than exceptionally powerful nuclear activity.

MacKenty (1989) compared the environments of 51 Seyfert galaxies to those of 51 "control" galaxies (chosen in a similar manner to those of Dahari). He found that $71 \%$ of the Seyferts had an apparent companion galaxy within 10 galaxy diameters vs. only $26 \%$ of the control sample. Since he did not explicitly correct for background galaxies, the true fraction of companions will be lower in both samples (presumably by similar amounts). He also found that a higher fraction of type 2 Seyferts had close companions than did type 1 Seyferts (however, the statistical significance level of this result is marginal). Finally, he showed that IR "colors" indicative of star-formation were preferentially associated with the Seyferts having close companions, and concluded that galaxy interactions may trigger excess star-formation in Seyferts (in accord with Dahari and DeRobertis).

Fuentes-Williams and Stocke (1988) measured the density of galaxies within $1 \mathrm{Mpc}$ of a sample of 53 Seyfert and 30 control galaxies. The galaxy density was quantitatively parameterized in several different ways, some of which were weighted strongly towards the nearest, brightest companions. Only galaxies with implied linear diameters between 15-50 kpc (corresponding to galaxies with absolute magnitudes near the fiducial Schecter $\mathrm{L}^{*}$ and comparable to those of the Seyferts themselves) were initially included as physical companions. No correction for the background was made. Unlike both Dahari and MacKenty, Fuentes-Williams and Stocke selected their control sample to match the Seyferts in Hubble type as well as absolute magnitude. They found no statistically-significant difference between the Seyfert and control samples in any of the quantities measuring local galaxy density. However, when apparent companion galaxies with diameters $<15 \mathrm{kpc}$ were included, the Seyferts did show statistically significant excesses of companions (though less dramatic than the excesses found by Dahari and by MacKenty).

Finally, Kollatschny and Fricke (1989) obtained spectra of 113 galaxies in 15 loose 
groups containing a Seyfert and 9 loose groups without. They found an excess of galaxies with strong emission-lines $\left(\mathrm{L}\left(\mathrm{H} \alpha>10^{41} \mathrm{erg} / \mathrm{sec}\right)\right.$ in the former compared to the latter groups (excluding the Seyfert galaxy itself). This is a fascinating result, but its connection to the triggering of Seyfert nuclei via galaxy interactions is not clear.

Can the results of Fuentes-Williams and Stocke be reconciled with those of the Dahari and Maclienty? There are several possibilities, some of which were discussed by FuentesWilliams and Stocke:

$>$ "Bad Luck" at the 2-3 $\sigma$ level.

$>$ There is a true excess of companions to Seyferts, but only for intrinsically faint companions.

$>$ The stronger excesses found by Dahari and MacKenty are (in part) artifacts of the way they defined a control sample. A random sample of field galaxies will tend to be more strongly weighted toward late-type galaxies than a sample of Seyferts (which are known to be primarily early-type spiral galaxies - cf. Simkin, Su, and Schwarz 1980). Since there is a well-known morphology-density relation such that galaxies of earlier Hubble type live preferentially in regions of higher galaxy density (Dressler 1980; Postman and Geller 1984), the Dahari/MacKenty control samples may be biased towards lower galaxy densities than their Seyfert samples.

$>$ The effect (excess of close companions) may be stronger for type 2 Seyferts than type 1's (MacKenty 1989; Petrosian 1982; Dahari and DeRcbertis 1988). If we include the type 1.5 Seyferts as type 1's and types 1.8 and 1.9 as type 2's, then the relative fraction of type 2 Seyferts in the samples of Fuentes-Williams and Stocke, MacKenty, and Dahari are 21\%, $28 \%$, and $57 \%$ respectively.

Since the pioneering work of Adams (1977), there have been several investigations of the morphology of Seyfert galaxies, most of which have noted the "surprisingly large" fraction of Seyferts with peculiar morphologies. This fraction ranges from $16 \%$ (Adams 1977 ) to $30 \%$ (MacKenty 1990) to $40 \%$ (Wehinger and Wyckoff 1977). The fraction of morphologically peculiar Seyferts seems to rise as the mean redshift of the sample under investigation rises. This may mean that the connection between galaxy interactions and the Seyfert phenomenon is strongest for the most luminous Seyfert nuclei. MacKenty also emphasized the significant fraction of Seyferts with amorphous morphologies, but colors too blue for $\mathrm{E}$ or S0 galaxies. He speculates that they may be post-merger/post-interaction galaxies (see also Hernquist 1989).

Simkin, Su, and Schwarz (1980) discussed images of the nearest Seyfert galaxies $(c z<5000 \mathrm{~km} / \mathrm{sec})$. They do not address the incidence of peculiar or interacting galaxies in this sample. Rather, they show that Seyferts can be comfortably accomodated within the standard Hubble/deVaucouleurs classification scheme, but that (compared to galaxies as a whole) the Seyferts are preferentially early-type spirals with inner and/or outer rings. They interpret these rings as signs of oval distortions in the disks, and speculate that such distortions lead to radial inflow of disk gas at a rate sufficient to fuel the nucleus. While they therefore favor an internal dynamical process for fueling the nucleus, galaxy interactions seem proficient at inducing bars/oval distortions in galactic disks (as reviewed elsewhere in this volume by Athanassoula).

ii) Seyfert Nuclei in Samples of Interacting Galaxies 
As in the types of investigations summarized above, there have been several wellconceived and carefully-executed spectroscopic surveys of the nuclei of interacting galaxies that have come to somewhat different conclusions concerning the incidence of Seyfert nuclei.

Keel et al. (1985) have compared the incidence rate of Seyfert nuclei in three samples: 1. a control sample consisting of 87 spiral galaxies in the Keel (1983) and Stauffer (1982) surveys of the nuclei of typical (non-interacting) galaxies 2. a complete sample of physical pairs of galaxies (61 galaxies) 3. 99 galaxies selected from the Arp Atlas to represent strongly interacting galaxies. The incidence rate of Seyferts was $5.6 \%$ in the control sample, $10.6 \%$ in the "pairs" sample, and $8.2 \%$ in the "Arp" sample. The excess of Seyferts in the "pairs" sample vs. the "control" sample is only marginally significant $(2 \sigma)$. If only the closest (separation < a galaxy radius) pairs are considered, the incidence rate of Seyfert nuclei rises to $25 \%$ (a $3 \sigma$ excess compared to the control sample). Because the galaxies in the "Arp" sample are more luminous (in the optical) than the galaxies in the control sample, and because the incidence rate of Seyfert nuclei increases with the optical luminosity of the host galaxy, Keel et al. argue that there is actually a deficiency of about a factor of two in the fraction of Seyfert nuclei in the "Arp" sample relative to a "luminosity-adjusted" control sample. Keel et al. also find that the Seyfert nuclei and LINER's in interacting galaxies may be slightly more luminous (on-average) than those in the control sample. Note that this does not agree with the results of Dahari and DeRobertis (1988).

Dahari (1985) obtained spectra of 167 galaxies selected from the Vorontsov-Vel'yaminov Atlas and Catalogue of Interacting Galaxies ("VV Catalogue"). He found that the fraction of Seyfert nuclei among the strongly interacting galaxies in the VV Catalogue (Interaction Class - "IAC" $=4,5$ or 6 ) was $13.2 \%$ vs. $5.6 \%$ for the weakly interacting (IAC = 2 or 3 ) VV galaxies vs. $4.6 \%$ for his control sample (the Keel + Stauffer noninteracting spirals). The excess of Seyferts in the strongly interacting VV sample compared to the control sample is significant at the $98 \%$ confidence level $(2.5 \sigma)$. While Dahari emphasizes a possible deficiency of Seyfert nuclei among the most strongly interacting VV galaxies (IAC $=6$ has no Seyferts among 14 galaxies), this is not a statistically significant result (only $88 \%$ confidence level). Note that Dahari has made not made a correction (analogous to the one applied by Keel et al. ) to account for possible differences in the absolute magnitudes of the galaxies in the control and VV samples.

Finally, Bushouse (1986) has obtained spectra of 94 disk galaxies selected from the Uppsala Galaxy Catalog (UGC) on the basis of morpholgical evidence for an interaction. He reports a deficiency of Seyfert nuclei in the interacting galaxies: $1 \%$ incidence rate vs. $5 \%$ in field spirals. However, I find that the statistical significance of the "deficiency" is only about $85 \%$ (when tested against the Keel+Stauffer control sample used by Dahari). Nevertheless his survey provides no evidence that interactions foster Seyfert nuclei.

Can all these results be made consistent? The simplest explanation is that (insofar as the neither the positive nor negative results are of overwhelming statistical significance) we are again being bedeviled by "bad luck" at the $2-3 \sigma$ level. It is also possible that the probability of detecting a Seyfert nucleus in an interacting galaxy is a complex function of the detailed properties of the interaction and of its constituent galaxies (as discussed 
by Byrd, Sundelius, and Valtonen 1987). In any case, it is difficult to ascribe the difference between Dahari and Keel et al. on the one hand and Bushouse on the other to any fundamental differences in either sample selection or observational approach. It therefore appears that samples of order 100 galaxies are not large enough to sort this out in a thoroughly convincing fashion (a depressing thought indeed).

\section{Radio Galaxies}

There have been myriad empirical investigations into the connection between radio emission from galaxies and their environments. I will limit the scope of the brief review to follow in two ways. First, I use the term "radio galaxy" only for cases in which the radio power at $1.4 \mathrm{GHz}$ is greater than about $10^{23} \mathrm{Watts} / \mathrm{Hz}$. My intent here is to exclude both galaxies in which the radio emission may be powered by a starburst (e.g. Condon etal. 1982) and typical Seyfert galaxies (e.g. Wilson and Heckman 1985) - both of which classes have been discussed above. Second, I will focus on environmental parameters that are the most likely to be related to galaxy interactions, namely the galaxy morphology and the local environment (within 100-200 kpc or so of the radio galaxy). In particular, I will not review the fascinating data concerning the membership of radio galaxies in rich clusters as a function of redshift (see, for example Prestage and Peacock 1988 and Yates, Miller, and Peacock 1990). Note that a somewhat expanded version of the following can be found in a recent review by Smith and Heckman (1990a).

Broadly speaking, there are two classes of radio galaxies (cf. Hine and Longair 1979 and Stocke and Perrenod 1981). The first class has weak optical emission-lines (usually LINER-type when classifiable) and the radio emission is dominated by emission from the jets with the brightness generally declining with distance from the nucleus (the so-called "FR I" radio morphology - after Fanaroff and Riley 1974). This class dominates the radio galaxy population for moderate radio powers $\left(\mathrm{P}_{1.4}=10^{23}-10^{25} \mathrm{Watts} / \mathrm{Hz}\right)$. The second class of radio galaxy has strong (generally Seyfert-like) optical emission-lines and the radio emission is dominated by the outer lobes/hotspots (a "classical double" or "FR II" radio morphology). This class dominates the radio galaxy population at high radio powers $\left(\mathrm{P}_{1.4}>10^{25}\right.$ Watts/Hz). I will henceforth use the terms "low-power" and "high-power" as a shorthand notation for the two respective classes.

\section{i) Low-Power Radio Galaxies}

There are many studies that have shown that the low-power radio galaxies are generically giant ellipticals (or related objects like cD galaxies) - see Smith and Heckman $(1989 \mathrm{a}, \mathrm{b})$ for a recent quantitative investigation of a large sample of such galaxies. That being the case, it is instructive to compare the local environments of low-power radio galaxies and optically similar, radio-quiet giant elliptical galaxies. Heckman, Carty, and Bothun (1985) conducted such an investigation using both the Palomar Sky Survey and CCD frames to measure the local galaxy density (within a projected radius of 100 $h^{-1} \mathrm{kpc}$ ) for samples of 47 radio-loud and 46 radio-quiet galaxies. They found that the low-power radio galaxies inhabit regions with average galaxy densities 2 to 3 times larger than the control sample (different at $4 \sigma$ level). Using a complementary approach that was less subject to subtle selection effects, Gavazzi and Jaffe (1986) searched for radio emission from nearly $300 \mathrm{E}$ and $\mathrm{S} 0$ galaxies in the Coma/A1367 supercluster. They found that 
the isolated $\mathrm{E}$ and $\mathrm{S} 0$ galaxies (no neighbors within $300 \mathrm{kpc}$ ) were low by a factor of 5 in radio power compared to the E's and S0's in denser environments. The Heckman, Carty, and Bothun and the Gavazzi and Jaffe papers together confirmed and extended the results in several important earlier papers - for example Dressel (1981) and Adams, Jensen, and Stocke (1980).

Smith and Hecliman (1989) and Heckman et al. (1986) found that low-power radio galaxies seldom show the type of structural peculiarities associated with galaxy interactions that involve one or more dynamically-cold systems (disk galaxies). That is, fewer than $10 \%$ exhibit "tails", "bridges", or "shells" having V-band surface brightnesses higher than 25 magnitudes per $\operatorname{arcsec}^{2}$. It is possible however that a greater fraction of such radio galaxies are strongly interacting with a neighbor (cf. Colina and Perez- Fournon 1990). This is because the radio galaxies themselves and their near neighbors tend to be earlytype galaxies (E's and S0's), and the morphological signatures of interactions between two such dynamically-hot systems can be quite subtle. Careful analysis may be required to distinguish tidal deformations from effects caused merely by overlapping the images of structurally normal galaxies (cf. Borne 1988; Lauer 1989). Moreover, since a large fraction of radio-quiet ellipticals show isophotal twists and nonconcentric isophotes (see the recent review by Kormendy and Djorgovski 1989), their mere presence in many low-power radio galaxies is not necessarily evidence that galaxy interactions are linked to the radio galaxy phenomenon. A rigorous comparison of the amplitude of such effects between radio-loud and radio-quiet ellipticals is needed, but has yet to be done. A possibly related effect is the claim by Bender and Möllenhoff (1987) that radio-loud ellipticals (what I would call low-power radio galaxies) tend to have "boxy" optical isophotes while radio-quiet ones tend to have "disky" isophotes. Neither the connection of boxy isophotes to past galaxy interactions nor the longevity of such structures is yet clear however (see Binney and Petrou 1985).

\section{ii) High-Power Radio Galaxies}

The relationship between galaxy interactions and high-power radio galaxies has only recently been addressed. This is largely because their low space density (and strong cosmological evolution) means that significant numbers of such objects are encountered only for $c z>10^{4} \mathrm{~km} / \mathrm{sec}$. The nearest high-power radio galaxy with strong optical emissionlines is the pathological object NGC1275. Other relatively local "famous" examples are 3C 120 and Cygnus $\mathrm{A}$. The large distances to these galaxies make them rather difficult to investigate optically. To give several examples of some of the more subtle difficulties: 1) The effects of the general background of faint galaxies need to be carefully and explicitly calculated before the galactic clustering environments of the radio galaxies can be determined 2) It is difficult to devise a suitable comparison or control sample (for better or worse, the morphologies and environments of the radio galaxies are usually compared to those of luminous elliptical galaxies in our local universe) 3) The radio galaxies often have large, bright, and morphologically complex emission-line nebulae (cf. Baum and Heckman 1989a,b; McCarthy 1989). Such a nebula can affect broad-band measurements of the properties of the stellar component of a radio galaxy (absolute magnitude, morphology, color), and so must be corrected for in some way. More rarely, a strong nonthermal (optical) nuclear source may be present and its effects accounted for. 
Heckman et al. (1986) published the results of an imaging survey of 43 (mostly highpower) radio galaxies. This sample was later enlarged to 72 galaxies with a median redshift of 0.06 by Smith and Heckman $(1989 a, b)$. The latter paper also presented color data (in the form of (B-V) global colors and color maps) for 56 of the radio galaxies. Hutchings (1987) and Yates, Miller, and Peacock (1990) have each discussed images of 25 more powerful and more distant (median $z 0.22$ and 0.31 respectively) radio galaxies. Smith and Heckman (1990b) have determined the local environment (within $100 h^{-1} \mathrm{kpc}$ ) of high-power radio galaxies, and have calculated the luminosity function of the associated galaxies (see also Fuentes-Williams and Stocke 1988). Finally, Smith, Heckman, and Illingworth (1990) have compared the stellar dynamical properties of high-power radio galaxies and normal giant elliptical galaxies. The principal results of these surveys are as follows:

$>$ Estimates of the fraction of high-power (strong-emission-line) radio galaxies that are morphologically distorted range from about 32\% (Yates, Miller, and Prestage 1990) to nearly 100\% (Hutchings 1987). Heckman et al. (1986) and Smith and Heckman (1989b) find that about half of this type of radio galaxy is morphologically- distorted at moderately high levels of surface brightness (brighter than $25 \mathrm{~V}$ magnitudes per $\operatorname{arcsec}^{2}$ in the radio galaxy rest-frame). The distortions they discuss take the form of tails, bridges to apparent companion galaxies, shells or fans, and strong dust lanes. In the great majority of their cases these are continuum-emitting structures (presumably starlight) and not merely emission-line gas. This is far greater than the incidence rate of such structures they found in the lower-powered radio galaxies with weak optical emission-lines (10\%), even though such features should have been more readily detectable in these latter galaxies because of their lower redshifts.

$>$ The high-power radio galaxies have (on-average) two bright companion galaxies ( $\mathrm{L}>0.2$ $\mathrm{L}^{*}$ ) within $100 \mathrm{kpc}$. While this may imply that interactions are occurring, this local density is no higher than that around typical radio-quiet ellipticals. However, the shape of the luminosity function of the companions differs from that of the standard Schecter function, having an enhanced relative number of bright galaxies. This might imply that interactions between massive galaxies can lead to strong radio emission.

$>$ The optical colors of the high-power radio galaxies are (on-average) several tenths of a magnitude bluer than those of either low-power radio galaxies or radio-quiet ellipticals of similar absolute magnitude. The two-dimensional color maps and radial color profiles show strong spatial variations, evidently reflecting the competing effects of dust and "young" ( $\ll 10$ Gigayear) stars.

$>$ The stellar dynamics of the radio galaxies with peculiar optical morphologies are also peculiar. The velocity dispersions are lower (by 0.1 dex on-average) and rotational support is more important (average $\mathrm{v}_{\mathrm{rot}} / \sigma$ greater by a factor of 2 to 3 ) than in radio-quiet ellipticals and morphologically-normal radio galaxies with similar absolute magnitudes.

The picture that emerges from these investigations is somewhat ambiguous. Strictly on the basis of morphology, the majority of powerful radio galaxies with strong emissionlines could be interpreted as mildly-damaged giant elliptical galaxies. That is, the structural peculiarities are transient and dynamically insignificant events that have not/will not change the Hubble type of the galaxy. This is the interpretation implicitly favored by Yates, Miller, and Peacock. The fact that many of these radio galaxies also have 
global colors that are significantly bluer than normal giant ellipticals could be explained by interaction-induced starbursts involving only a few percent mass of the "underlying" old (elliptical) galaxy (cf. Larson and Tinsley 1978). The excess blue light from a (post?) starburst population could explain the fact that the morphologically peculiar radio galaxies are about 1 magnitude brighter (at $\mathrm{V}$ ) than normal ellipticals with the same velocity dispersion (Smith, Heckman, and Illingworth 1990).

On the other hand, the rapid rotation that characterizes many of the morphologically peculiar galaxies is less easy to explain if these are just mildly damaged giant ellipticals. If the rapid rotators are the products of galaxy interactions or mergers, these must have been severe enough to affect the internal dynamics in a substantial way. Moreover, about one-third of the morphologically peculiar radio galaxies in Heckman et al. and Smith and Heckman appear likely (on morphological grounds) to be mergers or interactions involving two disk galaxies, because they exhibit two tail-like structures (cf. Toomre 1977). The clearest example of this is $3 \mathrm{C} 305$, and other possibly related radio galaxies are $3 \mathrm{C} 120$, 3C 171, 3C 223, 3C 285, 3C 382, 3C 459, PKS 1345+125, and PKS 2300-189.

At redshifts $z>0.5$ or so, the optical properties of high- power radio galaxies become even stranger. I have briefly described these galaxies in §IIIA above, and have argued there that the strong alignment between their optical and radio axes makes me skeptical that they can be simply interpreted as tidal interactions between galaxies (to paraphrase George Miley (1990) in his recent review of high-redshift radio galaxies, these galaxies are clearly "interacting", but probably not with another galaxy!). I will not discuss these fascinating objects further.

\section{iii) Gas Kinematics in Radio Galaxies}

The presence of appreciable interstellar gas in radio galaxies is often taken as evidence for an accretion event. This is particularly suggestive because the specific angular momentum of the gas is often much larger than (and sometimes misaligned with) the specific angular momentum of the stellar body of the galaxy (cf. Heckman et al. 1985 and references therein). The kinematics of the $\mathrm{H} I$ is the most illuminating, as van Gorkom et al. (1989) discuss. They have detected H I in absorption toward the nuclei of about $25 \%$ of a sample of low-redshift radio galaxies, and find the gas to be infalling. Since the detection of gas in absorption against a compact nuclear radio source requires a favorable viewing geometry, they suggest that most radio galaxies may have infalling HI. It is possible that the infalling $\mathrm{H} \mathrm{I}$ is material that has condensed out of a cooling flow, rather than tidally captured material. Maps of the $\mathrm{H} \mathrm{I}$ in emission are important in this regard, but will be very difficult to obtain.

D. QSO's

The observational and interpretational difficulties I described regarding the investigation of galaxy interactions involving high-power radio galaxies are even more severe in the case of QSO's. I will concentrate on low-redshift QSO's (at $z<0.5$ ), since our information concerning the host galaxies and environments of QSO's at higher redshifts is less complete.

i) Environments of QSO's

$>$ Radio-loud QSO's have more near-neighbors (within $100 h^{-1} \mathrm{kpc}$ ) than do typical galaxies by a factor of 4-5 (see Yee and Green 1984 and Smith and Heckman 1990b). 
In fact, the latter authors find an average of 2 physically-associated near-neighbors with $\mathrm{L}>0.2 \mathrm{~L}^{*}$. These results are intriguing, but their significance is clouded by the well-known morphology-density relation for galaxies. That is, while radio-loud QSO's have more nearneighbors than do galaxies as a whole, they do not differ significantly in this regard from radio-quiet giant elliptical galaxies. Thus, whether or not the radio-loud QSO's have an unusual number of near-neighbors depends on the nature of the appropriate comparison sample, which in turn depends on the (uncertain) nature of the QSO host galaxy.

$>$ Radio-quiet QSO's have fewer average near-neighbors than the radio-loud QSO's by a factor of 2 (Yee and Green 1984; Smith and Heckman 1990b). This means that they have about a factor of two more near-neighbors than average galaxies, but this result is only significant at the $2 \sigma$ level. Again, the nature of the appropriate comparison sample of normal galaxies is not entirely clear.

$>$ As in the case of the high-power radio galaxies (see above), the luminosity function of the near-neighbors of the radio-loud QSO's is unusually flat at the high luminosity end compared to a standard Schecter function (see Smith and Heckman 1990b). The redshifts of the QSO's under consideration are too low (typically 0.1 to 0.3 ) for this to have arisen due to the general evolution of typical galaxies. Moreover, Smith and Heckman find that the luminosity function of the near-neighbors of the radio-quiet QSO's is normal. The interpretation of this result is unclear, but one possibility is that it is a selection effect: if radio-loud QSO's and high- power radio galaxies are often triggered by the close encounter of two (or more) luminous galaxies, then the luminosity function of the near-neighbors will be biased towards the presence of such luminous galaxies.

$>$ While the environments of low- $z$ QSO's are locally dense, they are rarely located inside rich clusters of galaxies. Yee and Green (1984) suggest that QSO's are preferentially found in dense groups, and this suggestion is corraborated by the relatively small typical velocity differences between the QSO's and those near-neighbors with measured redshifts (cf. Heckman et al. 1984). This situation changes at $z>0.5$, where QSO's are often located in rich clusters (Yee and Green 1987).

$>$ The great majority (90\%) of the apparent near-neighbors to low- $z$ QSO's (galaxies projected to lie within $100 \mathrm{~h}^{-1} \mathrm{kpc}$ of the QSO) are in fact physically associated with the QSO because they have the same redshift (Heckman et al. 1984, and see also Stockton 1978).

\section{ii) Morphology of QSO Host Galaxies}

There have been several broad-band imaging surveys of low- $z$ QSO's that have emphasized the large fraction of QSO host galaxies that appear to be "disturbed" or "interacting": e.g. 35-55\% (Smith et al. 1986) and 30-40\% (Hutchings, Crampton, and Campbell,1984). The fraction of "disturbed/interacting" host galaxies may be particularly high for the radio-loud QSO's: 70\% (Hutchings 1987), 77\% (Smith et al. 1986), 50\% (Hutchings, Crampton, and Campbell 1984),> 68\% (Hutchings, Janson, and Neff 1989).

While these results are tantalizing, they do not yet constitute a water-tight case linking galaxy interactions to the QSO phenomenon. Some of the morphologically peculiar structures visible in the broad-band images of the radio-loud QSO's are likely to be produced by emission-line gas rather than starlight (for which there is considerable ambiguity in interpretation - see below). No one has yet made a rigorous effort to compare 
the fraction of "disturbed/interacting" QSO hosts to any realistic comparison sample of non-active galaxies (recall that the number of near-neighbors around low- $z$ QSO's may or may not be unusually large, depending on the Hubble type of the QSO host). For example, Lawrence et al. (1989) found that about $18 \%$ of optically-selected "field" galaxies with redshifts comparable to those of low- $z$ QSO's were classified by them as morphologically peculiar. While this fraction is significantly smaller than the fractions of "peculiar" QSO hosts quoted above, it is slightly worrying that the morphological classifications of the QSO and field galaxies were not made by the same people using the same data. At least we do have spectroscopic confirmation that many of the near-neighbors/interaction-partners are indeed galaxies at the same redshift of the QSO (see Stockton 1978 and Heckman et al. 1984).

Complimentary data have been published by Stockton and MacKenty (1987), who imaged 47 low redshift QSO's through narrow- band filters centered on the redshifted [O III] $\lambda 5007$ emission-line. They found that about $25 \%$ of these QSO's had luminous and highly structured emission-line nebulae with sizes of several tens of $\mathrm{kpc}$. They noted that these nebulae were preferentially associated with the QSO's having strong, spatiallyextended radio emission. They also emphasized that many of the QSO's with such nebulae were likely to be situated in interacting galaxies, and speculated that the nebulae might be gaseous tidal debris photoionized by the QSO.

An alternative interpretation of the QSO nebulae has been championed by Fabian and collaborators in a recent series of papers (cf. Crawford, Fabian, and Johnstone 1988 and references therein). They argue that the high densities inferred for the clouds in the nebulae require a high-pressure confining medium to be present. They therefore postulate that the visible nebulae are dense clouds photoionized by the QSO and imbedded in a hot $\mathrm{X}$-ray-emitting medium akin to the "cooling flows" associated with many cluster-dominant giant ellipticals. In their view the nebulae do not represent evidence for a tidal interaction. Moreover, Baum and Heckman (1988b) concluded that the nebulae were morphologically aligned with the axis of the radio source (as is the case for radio galaxies with similar radio properties). This raises the possibility that the morphology of the nebulae might be influenced by hydrodynamical processes associated with the outflowing radio plasma. Investigations of the dynamics of the gas might be able to discriminate between these various hypotheses, but most spectroscopy to date has been low-dispersion data with relatively poor velocity resolution (cf. Boroson, Persson, and Oke 1985; Crawford, Fabjan, and Johnstone 1988).

\section{CONCLUSIONS}

If nothing else, I hope this review has conveyed to the reader an impression of the amount of data, the number of different papers, and the multiplicity of approaches that deal with the question of the relationship between galaxy interactions and activity in galactic nuclei. To my mind there is little doubt that such a relationship exists. The more difficult question is whether galaxy interactions are the dominant mechanism by which nuclear activity is triggered. Somewhat surprisingly, the evidence seems to me to be in reasonable agreement with the theoretical/heuristic arguments summarized in $\S I I$ above.

I think the case is strongest for the luminous IR galaxies $\left(\mathrm{L}_{\mathrm{IR}}>10^{11} \mathrm{~L}_{\odot}\right)$. Over half of such galaxies have strongly peculiar optical morphologies. The redshifts of these galaxies 
are low enough that these morphological peculiarities can be compared to the results of simulated galaxy interactions, and the qualitative agreement is generally satisfactory. The high incidence rate of multiple nuclei in the most luminous such galaxies is very striking, because these are the very cases where the circumnuclear region is being subjected to maximal tidal stress. My only residual worry is that in some cases the peculiar structures visible in IR-luminous galaxies might have a hydrodynamical origin.

The QSO's and powerful radio galaxies with strong emission- lines (at $z<0.5$ where the optical/radio alignment effect is unimportant) are the next most convincing cases in my view. HST observations that more clearly delineate the optical morphology will be important (especially for the QSO's, where the amount of morphological information available in the present images is rather limited).

There is also suggestive evidence linking galaxy interactions to both the Seyfert and low-power radio galaxy phenomena. However (despite valiant efforts by several different groups working on large samples) this evidence remains at about the 2 to $4 \sigma$ level. This suggests to me that galaxy interactions are a significant, but probably not dominant, mechanism by which low-power nuclear activity is fuelled.

\section{REFERENCES}

Adams, T.F. 1977, Ap.J.Suppl., 33, 19.

Adams, M.T.,Jensen, E.B., and Stocke, J.T. 1980, Astr.J., 85, 1010.

Armus, L., Heckman, T.M, and Miley, G. 1987, Astr.J., 94, 831.

Armus, L., Heckman, T.M., and Miley, G.K. 1990, Ap.J., in press.

Baade, W., and Minkowski, R. 1954, Ap.J., 119, 206.

Balick, B. and Heckman, T.M. 1982, Ann.Rev.Astr.Ap., 20, 431.

Balzano, V.A. 1983, Ap.J., 268, 602.

Baum, S.A., and Heckman, T.M. 1989a, Ap.J., 336, 681.

Baum, S.A., and Heckman, T.M. 1989b, Ap.J., 336, 702.

Begelman, M., and Cioffi, D. 1989, Ap.J.Lett., 345, L21.

Bender, R., and Mollenhoff, C. 1987, Astr.Ap., 177, 71.

Binney, J., and Petrou, M. 1985, MNRAS, 214, 449.

Bome, K. 1988, Ap.J., 330, 38.

Boroson, T.A., Persson, S.E., and Oke, J.B. 1985, Ap.J., 293, 120.

Bothun, G., Lonsdale, C., and Rice, W. 1989, Ap.J., 341, 129.

Brinks, E. 1990, in Galaxy Dynamics and Interactions, ed. A. Toomre and R.Wielen, in press.

Bushouse, H. 1986, Astr.J., 91, 255.

Bushouse, H. 1987, Ap.J., 320, 49.

Bushouse, H., Lamb, S., and Werner, M. 1988, Ap.J., 335, 74.

Byrd, G., Sundelius, B., and Valtonen, M. 1987, Astr.Ap., 171, 16.

Campos-Aguilar, A., and Moles, M. 1990, preprint.

Carico, D., Graham, J., Matthews, K., Wilson, T., Soifer, B.T., Neugebauer, G., and Sanders, D. 1990, preprint.

Carter, D., Prieur, J., Wilkinson, A., Sparks, W.B., and Malin, D.M. 1988, MNRAS, 235, 813.

Chambers, K. 1989, Ph.D. thesis, The Johns Hopkins University. 
Chambers, K., Miley, G., and van Breugel, W. 1987, Nature, 329, 604.

Colina, L., and Perez-Fournon, I. 1990, Ap.J., 349, 45.

Condon, J, Condon, M., Gisler, G., and Puschell, J. 1982, Ap.J., 252, 102.

Crawford, C.S., Fabian, A., and Johnstone, R. 1988, MNRAS, 235, 183.

Cutri, R., and McAlary, C. 1985, Ap.J., 296, 90.

Dahari, O. 1984, Astr.J., 89, 966.

Dahari, O. 1985a, Astr.J., 90, 1772.

Dahari, O. 1985b, Ap.J.Suppl., 57, 643.

Dahari, O., and DeRobertis, M. 1988, Ap.J., 331, 727.

Djorgovski, S. 1987, in Starbursts and Galaxy Evolution, ed. T. Thuan, T. Montmerle, and J. Tran Thanh Van (Editions Frontieres: Paris), p. 401.

Dressel, L.L. 1981, Ap.J., 245, 25.

Dressler, A. 1980, Ap.J., 236, 351.

Fairclough, J. 1986, MNRAS, 219, 1P.

Fanaroff,B., and Riley, J. 1974, MNRAS, 167, 31P.

Franx, M., and Illingworth, G.D. 1988, Ap.J.Lett., 327, L55.

Fricke, K.J., and Kollatschny, W. 1989, in Proc. IAU Symp. 134, ed. D. Osterbrock and J.S Miller (Kluwer: Dordrecht), p. 425.

Fuentes-Williams, T., and Stocke, J. 1988, Astr.J., 96, 1235.

Gavazzi, G., and Jaffe, W. 1986, Ap.J., 310, 53.

Graham, J., Carico, D., Matthews, K., Neugebauer, G., and Soifer, B.T. 1990, preprint.

Gunn, J. 1979, In Active Galatio Nuclei, ed. C. Hazard, S. Mitton, p.213 (Cambridge University Press).

Heckman, T.M. 1983, Ap.J., 268, 628.

Heckman, T.M., Bothun, G.D., Balick, B., and Smith, E. 1984, Astr.J., 89, 958.

Heckman, T.M., Illingworth, G.D., Miley, G.K., and van Breugel, W. 1985, Ap.J., $299,41$.

Heckman, T.M., Armus, L., and Miley, G.K. 1990, Ap.J., in press.

Heckman, T.M., Carty, T., and Bothun, G. 1985, Ap.J., 288, 122.

Heckman, T.M., Smith, E., Baum, S., van Breugel, W., Miley, G., Illingworth, G., Bothun, G., and Balick, B. 1986, Ap.J., 311, 526.

Hernquist, L. 1989, Nature, 340, 687.

Hickson, P., Menon, T., Palumbo, G., and Persic, M. 1989, Ap.J., 341, 679.

Hine, R., and Longair, M. 1979, MNRAS, 188, 111.

Hummel, E. 1981, Astr.Ap., 96, 111.

Hunter, D., Gillet, F., Gallagher, J., Rice, W., and Low, F. 1986, Ap.J., 303, 171.

Hutchings, J. 1987, Ap.J., 320, 522.

Hutchings, J., Crampton, D., and Campbell, B. 1984, Ap.J., $280,41$.

Hutchings, J., Janson, T., and Neff, S. 1989, Ap.J., 342, 660.

Hutchings, J., and Neff, S. 1988, Astr.J., 96, 1575.

Jedrzejewski, R., and Schecter, P. 1988, Ap.J.Lett., 330, L87.

Joseph, R., and Wright, G. 1985, MNRAS, 214, 87.

Joseph, R., Meikle, W., Robertson, N., and Wright, G. 1984, MNRAS, 209,111.

Keel, W.C. 1983, Ap.J.Suppl., 52, 229.

Keel, W.C. 1987, in Proc. IAU Symp. 121, ed. K.J. Fricke, E. Khachikian, and J. Melnick. 
Keel, W., Kennicutt, R., Hummel, E., and van der Hulst, J. 1985, Astr.J., 90, 708.

Kennicutt, R., Keel, W., van der Hulst, J., Hummel, E., and Roettiger 1987, Astr.J., 93, 1011.

Kollatschny, W., and Fricke, K. 1989, Astr.Ap., 219, 34.

Kormendy, J., and Djorgovski, S. 1989, Ann.Rev.Astr.Ap., 27, 235.

Larson, R. 1987, in Starbursts and Galaxy Evolution, ed. T. Thuan, T. Montmerle, and

J. Tran Thahn Van (Editions Frontieres: Paris), p. 467.

Larson, R., and Tinsley, B. 1978, Ap.J., 219, 46.

Lauer, T. 1988, Ap.J., 325, 49.

Lawrence, A., Rowan-Robinson, M., Leech, K., Jones, D., and Wall, J. 1989, MNRAS, $240,329$.

Lonsdale, C., Persson, S.E., and Matthews, K. 1984, Ap.J., 287, 95.

MacKenty, J. 1989, Ap.J., 343, 125.

MacKenty, J. 1990, preprint.

McCarthy, P. 1988, Ph.D. thesis, University of California, Berkeley.

McCarthy, P., van Breugel, W., Spinrad, H., and Djorgovski, S. 1987, Ap.J.Lett., 321, L29.

Meixner, M., Blitz, L., Puchalsky, R., Wright, M., and Heckman, T. 1990, Ap.J., in press.

Melnick, J. 1987 in Starbursts and Galaxy Evolution, ed. T. Thuan, T. Montmerle, and J. Tran Thanh Van (Editions Frontieres:Paris), p. 215.

Miley, G.K. 1990, in Galaxy Dynamics and Interactions, ed. A. Toomre and R.Wielen (in press).

Noguchi, M. 1988, Astr.Ap., 203, 259.

Petrosian, A.R. 1982, Astrofizika, 18, 548.

Postman, M., and Geller, M. 1984, Ap.J.. 281, 95.

Prestage, R., and Peacock, J. 1988, MNRAS, 230, 131.

Rees, M. 1984, Ann.Rev.Astr.Ap., 22, 471.

Rieke, G.H. 1988, Ap.J.Lett., 331, L5.

Salzer,J. 1987, Ph.D. thesis, University of Michigan.

Sanders, D., Soifer, B.T., Elias, J., Madore, B., Matthews, K., Neugebauer, G., and Scoville, N. 1988a, Ap.J., 325, 74.

Sanders, D., Scoville, N., Sargent, A., and Soifer, B.T. 1988b, Ap.J., 324, L55.

Schweizer, F., and Seitzer, P. 1990, preprint.

Simkin, S.M., Su, H.J., and Schwarz, M.P. 1980, Ap.J., 237, 404.

Smith, E., Heckman, T., Bothun, G., Romanishin, W., and Balick, B. 1986, Ap.J., 306, 64.

Smith, E., and Heckman, T. 1989a, Ap.J.Suppl., 69, 365.

Smith, E., and Heckman, T. 1989b, Ap.J., 641, 358.

Smith, E., and Heckman, T. 1990a, Ap.J., in press.

Smith, E., and Heckman, T. 1990b, in Galaxy Dynamics and Interactions, ed A. Toomre and R. Wielen (in press).

Smith, E., Heckman, T., and Illingworth, G. 1990, Ap.J., in press.

Soifer, B.T., Houck, J.R., and Neugebauer, G. 1987, Ann.Rev.Astr.Ap., 25, 187.

Stauffer, J. 1982, Ap.J.Suppl., 50, 517.

Stocke, J.T. 1978, Astr.J., 83, 348. 
Stocke, J.T., and Perrenod, S. 1981, Ap.J., 245, 375.

Stockton, A. 1978, Ap.J., 223, 747.

Stockton, A. 1990, in Galaxy Dynamics and Interactions, ed. A. Toomre and R. Wielen (in press).

Stockton, A., and MacKenty, J. 1987, Ap.J., 316, 584.

Sulentic, J.W. 1976, Ap.J.Suppl., 32, 171.

Sulentic, J.W. 1990, preprint.

Telesco, C. 1988, Ann.Rev.Astr.Ap., 26, 343.

Toomre, A. 1977, in Evolution of Galaxies and Stellar Populations, ed. B.M. Tinsley and R.B. Larson, p.401 (Yale University Observatory).

Toomre, A., and Toomre, J. 1972, Ap.J., 178, 623.

van Albada, T., Kotanyi, C., and Schwarzchild, M. 1982, MNRAS, 198, 303.

van Gorkom, J., Knapp, G., Ekers, R., Ekers, D., Laing, R., and Polk, K. 1989, Astr.J., 97,708 .

Wehinger, P., and Wyckoff, S. 1978, MNRAS, 184, 335.

Williams, R.E., and Christiensen, W. 1985, Ap.J., 291, 80.

Wilson, A.S., and Heckman, T.M. 1985, in Astrophysics of Active Galaxies and QSO's, ed. J. Miller (University Science Books:Mill Valley, CA).

Yates, M.G., Miller, L., and Peacock, J.A. 1990, MNRAS, in press.

Yee, H., and Green, R. 1984, Ap.J., 280, 79.

Yee, H., and Green, R. 1987, Ap.J., 319, 28. 
G. Burbidge: I believe that your point that seyfert galaxies are found in regions of higher galaxy density than average is very important. The same result is found for low-z QSOs, as you said. It is also found in the situation where the QSOS and galaxies have very different red shifts, based on a very large sample of QSO-galaxy pairs studied in Burbidge, Hewitt, Narlikar, and Das Gupta. There are also reports that the same result is being found by Hammer and his colleagues for high redshift radio galaxies. Thus a general rule is emerging. Whereever we see a non-thermal source it is most likely with it has been generated in a region of high galaxy density. Since gravitational microlensing will not account for the QSO-galaxy pairs where $\mathrm{Z}_{\mathrm{G}}<$ $\mathrm{z}_{\mathrm{O}}$, we are then forced to the conclusion that the non-thermal source is ejected from "normal" galaxies.

Kochhar: You said that tidal interactions do not seem to be very important in determining seyfert activity. What role do internal dynamics play? (After all, spirals already have gas, and all they need is a way of channeliing it to the center, unlike ellipticals which need gas.)

T. Heckman: The interesting paper by Simkin, Su, and Schwarz suggested (on the basis of the optical morphology) that internal dynamical processes were very important in fueling seyfert nuclei. There is still not enough dynamical data to corroborate this idea.

Neff: Comment: Many seyferts show signs of previous interactions when studied carefully.

Osterbrock: To me the statistical evidence that a surplus of galaxies undergoing interactions are seyferts, and that a surplus of Seyferts (compared with other galaxies) are undergoing interactions is quite persuasive. Doubtless there are additional cases of interactions not directly counted as galaxies with nearby "neighbors" or "companions"---cases in which the perturbing galaxy is below the magnitude limit, cases in which the perturber is not seen because of projection effects, and cases in which the perturber has already merged with the galaxy it perturbed. That a large fraction of the perturbers are dwarf galaxies can be understood from the fact that a large fraction of galaxies are dwarfs.

Heckman: I agree that the existing investigations may well underestimate both the fraction of seyferts undergoing interactions and the fraction of interacting galaxies that have Seyfert nuclei. Nevertheless, careful studies by independent authors have reached conflicting results. This suggests (to me) that the seyfert/interaction connection is subtle, complex, and (possibly) rather weak. 